\title{
Web-based Education for All: A Tool for Development Adaptive Courseware
}

\author{
Peter Brusilovsky \\ School of Computer Science \\ Carnegie Mellon University \\ Pittsburgh, PA 15213 \\ plb@cs.cmu.edu \\ John Eklund \\ Faculty of Education \\ The University of Technology \\ Sydney NSW Australia \\ j.eklund@uts.edu.au \\ EImar Schwarz \\ Computer Science Department \\ University of Karlsruhe \\ Germany \\ ukgo@rz.uni-karlsruhe.de
}

\begin{abstract}
Many Web-based educational applications are expected to be used by very different groups of users without the assistance of a human teacher. Accordingly there is a need for systems which can adapt to users with very different backgrounds, prior knowledge of the subject and learning goals. An electronic textbook is one of the most prominent varieties of Web-based educational systems. In this paper we describe an approach for developing adaptive electronic textbooks and present InterBook an authoring tool based on this approach which simplifies the development of adaptive electronic textbooks on the Web.
\end{abstract}

Keywords: electronic textbook, adaptive navigation support, Web-based education, student model, adaptive hypermedia

\section{Web-based Education}

\subsection{The Case for Adaptation}

While many research papers and media publications report substantial success with Web-based education, a careful analysis of the situation and informal discussions with "on-line teachers" show that Web-based education is quite far from achieving its main goal - reaching a wide distance audience in a cost effective And educationally sound manner, and in particular accommodating the requirements of students from underrepresented groups. Most of the positive 
experiences show that Web-based education works well for well-prepared, well-motivated students in reasonably homogeneous virtual classrooms. An example of a successful area are the masters-level courses for distance graduate students, certificate programs for students with college degrees, and various Web-based courses for sophomore/senior level students who take the course from the same or a remote campus. Web-based teaching of freshman and junior level students who are less prepared and require a lot of attention from a teacher is more difficult. Currently the use of Computer Mediated Communication (CMC) tools to deliver instruction works well for in-campus students, who can visit teachers and assistants in office hours. The worst case is freshman and junior level distance students, who often are supposed to be the main audience for future Web-based education. Current experience shows that distance education works here only when teachers and assistants can afford a lot of time for Web-based, e-mail, or telephone communication with their students (note that any Internet communication is still more time-consuming than real-time phone communication). This is not very encouraging. Current "pioneer" distance students are still special - those who are already reasonably well prepared and motivated to register for a Web-based course, and for whom distance is the only problem (an example is high schools students earning their first credits). What needs to be changed in the current model of Web-based distance education to make it available for less prepared and less motivated students?

We think that one of the difficulties with Web-based education is the course material which currently comes in various forms - lectures, tutorials, examples, quizzes, and assignments. In many current Web-based courses, the course material is still implicitly oriented for a traditional on-campus audience - reasonably homogeneous, reasonably well-prepared and well-motivated students who have access to teachers and assistants to fill possible gaps and resolve misunderstandings. However, Web-based courses are to be used by a much wider variety of users than any campus-based courses. These learners may have very different goals, backgrounds, knowledge levels, and learning capabilities. A Web-based course which is designed with a particular class of users in mind (as it is usually done for on-campus courses) may not suit other users. The only way to enhance it is to make the course material richer and more flexible so that different students can get personalized content and a personalized order of presentation. Current Web-based courses in this respect are not "flexible". In the best case, course material is a network of static hypertext pages with some media enhancement. Neither the teacher nor the delivery system can adapt the course presentation to different students. As a result, some students waste their time learning irrelevant or already known material, and some students fail to understand (or just misunderstand) the material and consequently overload a distance teacher with multiple questions and requests for additional information.

Our suggestion is to develop Web-based courses which can adapt to users with very different backgrounds, prior knowledge of the subject and learning goals. For example, students with specific goals could get some parts of the course in more detail, students with some knowledge of the subject would not be taught the known material again, and less prepared students would get more examples and more problems, starting from the very simple.

We distinguish three levels or steps of increasing complexity on the way to a flexible and adaptive courseware. The first level is to provide a well-designed hypermedia-based course containing all the required educational material: explanations, examples, tests, and problems for students with different learning abilities. The hypermedia interface makes it theoretically possible for each student to find his or her unique path through the hyperspace of learning material. This 
step could be made in many Web-based courses within the existing framework. However, the first level is not enough for less-prepared students such as many college and university freshman and distance students. These users who have never worked with complex information systems simply cannot cope with advanced hyperspaces offered by modern Web-based applications. They will rarely be able to find a hyperspace path which is most relevant to their knowledge, background and goals. The second step is to let teachers tune a generic course to the needs of particular students and classes by specifying a subset of topics to learn and the order of learning. The third step is to provide adaptive guidance mechanisms specially tailored to accommodate the current knowledge, learning goals, and information seeking tasks of that individual user. Guidance in this context addresses the problem of a users' unproductive wandering, refocussing them on their learning objectives, suggesting logical next steps to inform them about the knowledge structure of the hyperspace, or re-sequencing materials according to their demonstrated knowledge of content. In these cases, the knowledge implicit in the hypermedia is well defined and carefully structured, similar to that found in a textbook. Adaptive guidance is especially important for Web-based courses because in many cases the user is "alone" working with it (probably from home). That is why "external" guidance, in which a colleague or a teacher typically provides adaptively in a normal classroom situation, is not available. We claim that all three steps up to the level of adaptive guidance must be made to turn Web-based education into a way to maximize educational opportunity for every type of learner.

\subsection{Adaptive Web-based education}

A number of research groups worldwide are working now on adaptive Web-based applications driven by the importance of adaptivity in the context of Web-based education (Brusilovsky, Nakabayashi \& Ritter, 1997). Currently, two adaptive hypermedia technologies were used for providing adaptive Web-based course material: adaptive presentation, and adaptive navigation support.

Adaptive navigation support (ANS) is aimed to help students to find an "optimal path" through the hyperspace of learning material. The most popular forms of ANS on the Web are direct guidance, annotation, and disabling. Direct guidance was implemented in different forms in the following Web-based systems: ELM-ART-II (Weber \& Specht, 1997), CALAT (Nakabayashi et al., 1997), InterBook (Brusilovsky, Eklund \& Schwarz, 1997), AST (Specht et al., 1997), Medtec (Eliot, Neiman \& Lamar, 1997), and DCG (Vassileva, 1997). Annotation was implemented in InterBook (Brusilovsky, Eklund \& Schwarz, 1997), WEST-KBNS (Eklund \& Sawers, 1996), ELM-ART (Brusilovsky, Schwarz, \& Weber, 1996), and AST (Specht et al., 1997). Disabling was implemented in 2L670 (Calvi \& De Bra, 1997).

Adaptive presentation is aimed to adapt the page content to knowledge, goals, and other characteristics of an individual user. It is very important in WWW context where the same "page" has to suit to very different students. Only two systems implement full-fledged adaptive presentation: C-Book (Kay \& Kummerfeld, 1994) and 2L670 (Calvi \& De Bra, 1997).

\subsection{The problem of authoring}

One problem of all existing adaptive Web-based systems is that their development is quite time consuming. Until recently, any Web-based courseware development was time-consuming. Currently, a great variety of tools for developing Web-based courses is available. In addition to small specialized tools which are suitable for developing one of the course components 
mentioned above, there are many integrated tools which supports development of all course components. Here are just a few most well-known examples of university developed tools: WebCT (Goldberg, Salari \& Swoboda, 1996), Virtual-U (Harasim, Calvert \& Groenboer, 1997), CyberProf (Hubler \& Assad, 1995), Gentler (Thimbleby, 1997), Albatros (Lai, Chen \& Yuan, 1995), FLAX (Routen, Graves \& Ryan, 1997), and Mallard (Graham \& Trick, 1997). A number of tools originally developed in universities are now commercially available (CourseInfo, 1997; Docent Software Inc., 1997; Interactive Factory, 1997; liveText Publishing, 1997; madDuck Technologies, 1997). The most well-known commercial tools, TopClass (WBT Systems, 1997) and LearningSpace (Lotus, 1997), are quite mature and used in many universities. The problem with all the above tools is that none of them supports development of truly adaptive courseware. TopClass (WBT Systems, 1997) is capable of annotating, on an individual basis, folders as "unread" if they contain unvisited pages, or "new" if they contain new material. In this sense, and according to the definition of an adaptive hypermedia system, it is correct to describe TopClass as a weakly adaptive environment offering link annotation in a simple form. However, in this case there is no domain structure, that is, no indication of how information embedded in the pages is related. The rest of the paper describes InterBook, a tool for developing adaptive Web-based courseware. We present the approach behind the tool and provide some information concerning interface, implementation, and evaluation.

\section{InterBook: The Approach}

InterBook is based on a specific concept-based approach suggested originally in (Brusilovsky, 1995) and further elaborated by the ELM research group in the process of development an adaptive Web-based LISP textbook ELM-ART (Brusilovsky, Schwarz \& Weber, 1996). ELMART happens to be one of the first and most influential adaptive educational system on the Web. A number of existing Web-based adaptive systems are based on the ELM-ART architecture. InterBook is an authoring system which implements this approach. The current metaphor behind the InterBook approach is an electronic textbook (ET) which can be any hierarchically structured hypermedia material. An electronic textbook is also one of the most popular metaphors for representing online course material. Virtually any kind of course material can be represented as an electronic textbook. This section presents the InterBook approach and the current interbook system in some detail.

\subsection{Content representation}

The InterBook approach uses two kinds of knowledge: knowledge about the domain being taught (represented in the form of a domain model) and knowledge about the students (represented in the form of individual student models). The domain model serves as a basis for structuring the content of an adaptive ET. We distinguish two content parts in an adaptive ET: a glossary and a textbook (or a shelf of textbooks).

The domain model and the student model. According to the InterBook approach, the keys to adaptivity in an adaptive ET are the domain model and the student model. The simplest form of domain model is just a set of domain concepts. What we call concepts are named differently in different research papers - topics, knowledge elements, objects, learning outcomes, but in all cases they are just elementary pieces of knowledge for the given domain. Depending on the domain and the application area, concepts can represent bigger or smaller pieces of domain 
knowledge. A more advanced form of the domain model is a network, with nodes corresponding to domain concepts and links reflecting several kinds of relationships between concepts. This network represents the structure of the domain covered by a hypermedia system. The domain model provides a structure for representation of the student's knowledge of the subject. For each domain model concept, an individual student's knowledge model stores some value which is an estimation of the student knowledge level of this concept. This type of model (called an overlay model) is powerful and flexible: it can independently measure the student's knowledge of different topics. The overlay student model can be kept up-to-date relatively easy. All student actions (page visits, problem-solving, quizzes answering) are tracked and used to increase or decrease knowledge levels for involved concepts. Another component of the student model is the model of student's learning goals. Each student may have an individually assigned learning goal. A learning goal is just a set of concepts to be learned. A sequence of assigned learned goals forms an individual order of learning. Adaptive guidance mechanisms will ensure that the student achieves the first learning goal in a sequence, then the second one, and so forth. An individual order of learning is a convenient interface for the teacher to specify an individual syllabus for a student or a class. It may be done to adapt to special goals or backgrounds of the students, or to a particular textbook. An individual order of learning plus adaptive guidance are the mechanisms which are able to build an unlimited number of personalized adaptive courses from the same course material.

The glossary. The glossary is the central part of the ET. According to our approach, the glossary is considered as a visualized (and externalized) domain network. Each node of the domain network is represented by a node of the hyperspace, while the links between domain network nodes constitute main paths between hyperspace nodes. The structure of the glossary resembles the pedagogical structure of the domain knowledge and, vise versa, each glossary entry corresponds to one of the domain concepts. The links between domain model concepts constitute navigation paths between glossary entries. Thus, the structure of the glossary resembles the pedagogic structure of the domain knowledge. In addition to providing a description of a concept, each glossary entry provides links to all book sections which introduce or require the concept (Figure 1). This means that the glossary integrates traditional features of an index and a glossary.

The indexed textbooks. InterBook represents educational material as a set of Electronic Textbooks. A human-written ET represents human teaching expertise on how to introduce the domain concepts to the learners. It is usually a real textbook represented in hypermedia form. Each ET is hierarchically structured into units of different level: chapters, sections, and subsections. Each terminal level unit is an atomic presentation, example, problem, or test. Several ET on the same subject form a bookshelf. To make an ET "more intelligent" and to connect it to the glossary, we have to let the system know what each unit of the textbook is about. This is done by indexing of textbook units with domain model concepts. All books from the same bookshelf are indexed with the same set of domain model concepts. InterBook uses role-based indexing (Osin, 1976). Each terminal unit has an attached list of related concepts (we call this list spectrum of the unit). For each involved concept, the spectrum represents the name and the role of the concept in the unit. Currently we support two roles: each concept can be either a outcome concept or a prerequisite concept. A concept is included in the spectrum as a outcome concept if some part of this unit presents the piece of knowledge designated by the concept. A concept is 
included in the spectrum as a prerequisite concept if a student has to know this concept to understand the content of the unit. Part of the InterBook approach is visualization of unit indexes, i.e., showing the set of outcome and prerequisite concepts of a unit on a concept bar to the right of the content of the unit (Figure 2).

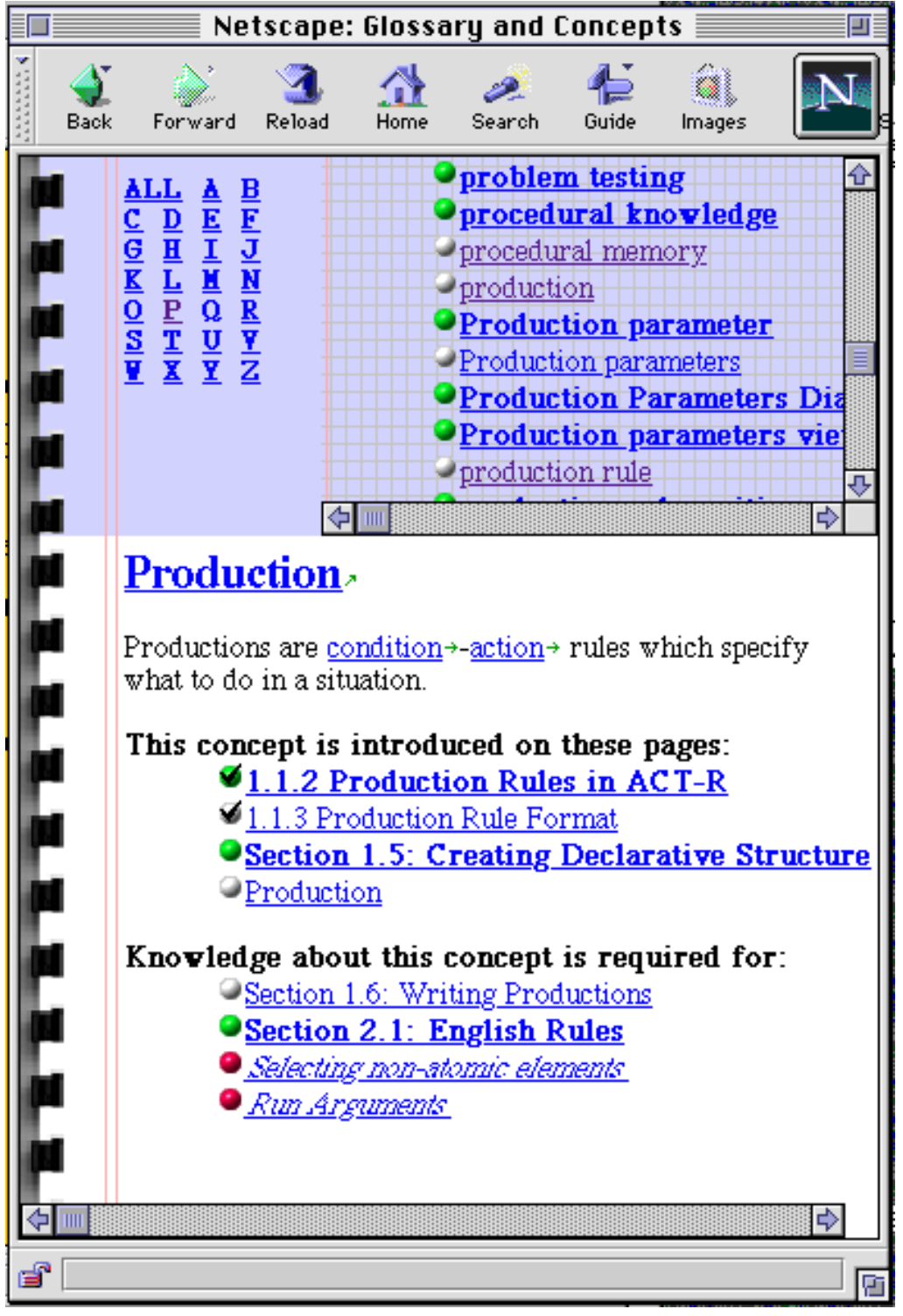

Figure 1: A glossary window showing a "page" for production.

\subsection{Functionality}

Domain model-based indexing is a relatively simple but powerful mechanism, because it provides the system with knowledge about the content of its pages: the system knows which concepts are presented on each page and which concepts have to be learned before starting to learn each page. It opens the path for several adaptation techniques presented in this subsection. 


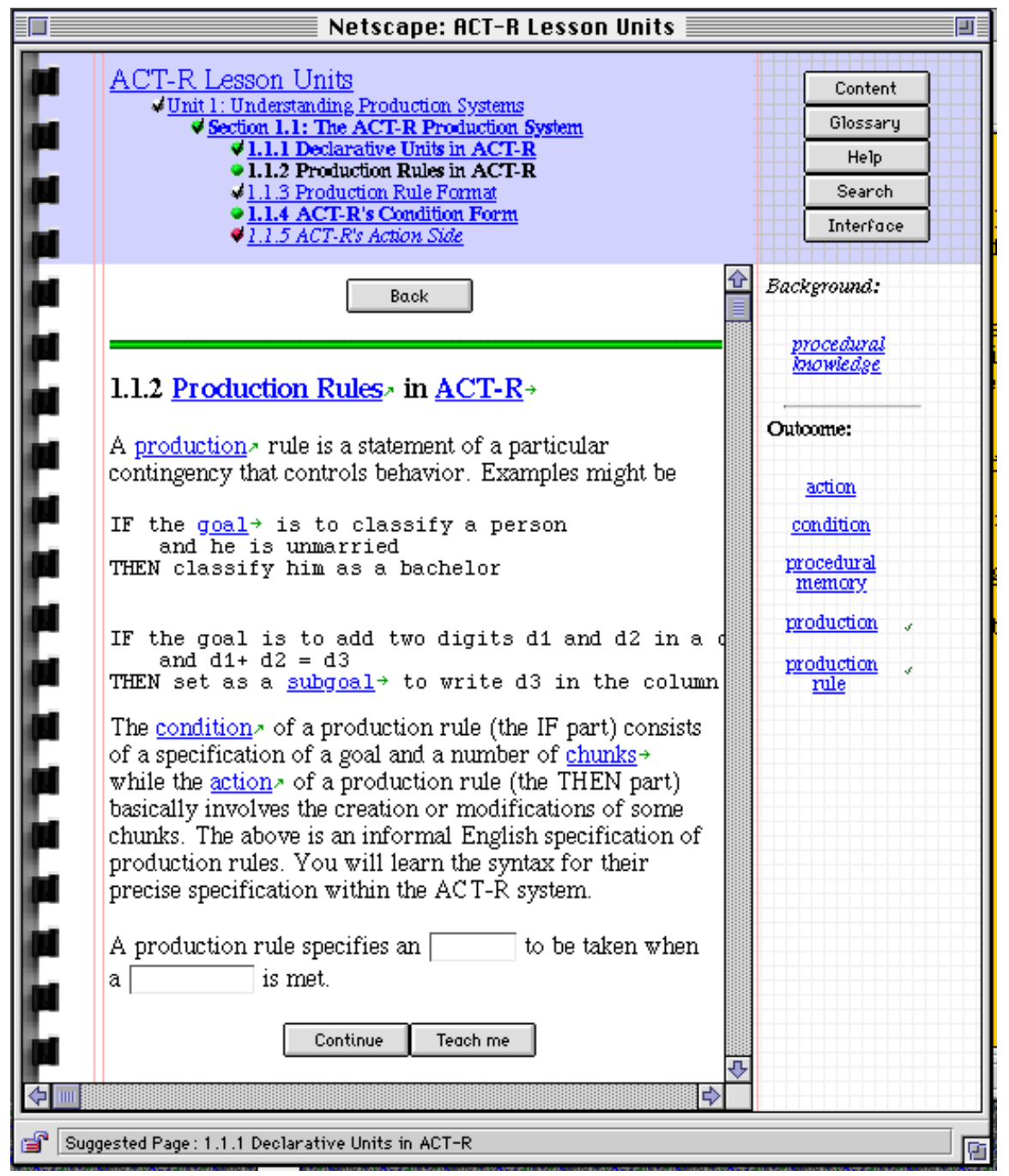

Figure 2: A section of ACT-R course material as it is presented by Netscape browser. The Concept bar (left) shows outcome and background concepts for the section. The Navigation center (top) let the user move in one click to any section on the same or upper level. Colored balls (up) and checkmarks (right, on the concept bar) provide adaptive annotation. The button "Teach me" provides direct guidance.

Advanced navigation. The knowledge about the domain and about the textbook content is used to serve a well-structured hyperspace. The system provides all regular navigation tools: sequential (forward/backward) and hierarchical (up/down) links within unit hierarchy, provides the navigation center for one-click transfer to all sections on the same or upper levels, and generates a table of contents where all entries are clickable links. In addition, it generates links between the glossary and the textbook. The concept bar provides links from each textbook unit to corresponding glossary pages for each involved concept (Figure 2). On the other hand, from each glossary page describing a concept the system provides links to all textbook units which can be 
used to learn this concept (Figure 1). These links are not stored in an external format but generated on-the-fly by a special module which takes into account the student's current state of knowledge represented by the student model. This approach not only reduces page design time, but also provides the opportunity for direct guidance, adaptive navigation support, and prerequisite-based help.

Direct guidance and adaptive navigation support. The InterBook approach provides many more opportunities for browsing the course materials than traditional on-line textbooks. The negative side of this is that there is a higher risk for the student to get lost in this complex hyperspace. To support the student navigating through the course, the system uses adaptive annotation and direct guidance technologies. Adaptive annotation means that the system uses visual cues (icons, fonts, colors) to show the type and the educational state of each link. Direct guidance means that the system can suggest to the student the next part of the material to be learned. Using the student model, it is possible to distinguish several educational states for each unit of ET: the content of a unit can be known to the student, ready to be learned, or not ready to be learned (the latter example means that some prerequisite knowledge is not yet learned). The icon and the font of each link presented to the student are computed dynamically from the individual student model. They always inform the student about the type and the educational state of the unit behind the link. In InterBook, red means not ready to be learned, green means ready and recommended, and white means no new information. A checkmark is added for already visited units (Figures 1 and 2). The same mechanism can be used to distinguish and show several levels of students knowledge of the concepts shown on the concept bar. In InterBook, no annotation means "unknown", a small checkmark means "known" (learning started), a medium checkmark means "learned" and a big checkmark means "well-learned" (Figure 1). For many students, adaptive guidance provides enough support to make a navigation decision. Those who hesitate to make a choice could push the button "Teach me" and the system will apply several heuristics to select the most suitable node among those ready to be learned.

Prerequisite-based help. Prerequisite-based help is yet another adaptive guidance technique which is problem-driven rather than goal-driven. The system's knowledge about the course material comprises knowledge about what the prerequisite concepts are for any unit of the textbook. Often, when students have problems with understanding some explanation or example or solving a problem, the reason is that some prerequisite material is not understood well. In that case they can request prerequisite-based help (using a special button), and the system generates a list of links to all sections which present some information about background concepts of the current section. This list is adaptively sorted according to the student's knowledge as represented in the student model: more "helpful" sections are listed first. Here "helpful" means how informative the section is to learn about the background concepts. For example, the section which presents information about an unknown background concept is more informative than a section presenting information about a known concept. A section which presents information about two unknown background concepts is more informative than a section presenting information about one concept. Prerequisite-based help is a very suitable technique to support student-driven learning. Students may have different interests in the same course and often jump far ahead in the course to reach the goal part of the material as far as possible. For these students, prerequisite-based help will support backward learning mode, i.e., starting from the goal portion of the course, learn the minimum background knowledge required to understand the goal part. 


\section{InterBook Aspects}

This sections presents several aspects of InterBook: interface, implementation, and authoring. These aspects are secondary to the adaptive approach implemented in the Interbook. We can envision different interface and different implementations. In particular, authoring in InterBook could be significantly improved. At the same time, the information provided below is essential to complete a description of InterBook.

\subsection{Interface}

InterBook uses advanced features of modern browsers such as multiple windows and frames to provide the student with useful and powerful interface. Main windows used by InterBook are the textbook window (Figure 2) and the glossary window (Figure 1).

The Glossary window is used to view the glossary. The upper part of the window is a list of glossary concepts. The lower part of this window is used to show the glossary entry for a concept. For each concept the system presents the concept description (if provided by the author), the list of section titles (selected from all available textbooks) which present the concept (i.e., which have it as an outcome concept) and the list of section titles which require this concept (i.e., which have it as a background concept). Section titles are clickable links which makes the corresponding section to be loaded to the Textbook window.

The Textbook window is the most important window in InterBook interface. This window is designed to view the main content of a textbook, section by section. It is divided into frames performing different functions. Main frame of the Textbook window is the Text window. This window shows a particular section of the textbook which is called current section. For a terminal section the Text window shows the title of the section and the section itself. For a high-level section the Text window shows the title, the section preface (if existing) and the full table of content for the section (i.e. list of hierarchically structured titles of its subsections down to terminal level). A vertical bar to the right of the Text window is the Concept bar. It is used to show the concepts related with the current section. All names of concepts on the Concept bar are links to the Glossary. The upper part of the Textbook window hosts the navigation center and the toolbox. The navigation center shows the position of the current section in the textbook: it lists the titles of all direct predecessors ("father", "grandfather", etc.) and all "brothers" of the current section. All names of the sections are clickable links. The navigation center serves for both orientation and navigation. The toolbox provides a set of buttons which are used to call additional windows (such as content window, search window, and prerequisite-based help window) which provides additional functionality.

\subsection{Implementation}

The WWW implementation of InterBook is based on the Common Lisp Hypermedia Server CLHTTP (Mallery, 1994). CL-HTTP is a fully featured HTTP server completely implemented in Common LISP. CL-HTTP appears to be an optimal platform for our purposes. CL-HTTP offers a Common Gateway Interface to handle incoming URLs. To enable the server to respond to a particular URL, this URL has to be associated to a response function implemented in LISP. Answering an incoming request, the server recognizes a URL, calls an associated function, and passes the received URL and enclosed form values as parameters. In response, the function generates an HTML page. To do that, it can use a comprehensive library of HTML-generating 
functions. With such an architecture, CL-HTTP is a very flexible and powerful tool for implementing intelligent applications on WWW. Since a LISP function is called to handle the request, any interactive or intelligent tool written in LISP can be connected to WWW with the help of CL-HTTP.

\subsection{Authoring}

Authoring an adaptive electronic textbook can be divided into 5 steps which are described in detail below (see Figure 3). In brief, an Electronic Textbook is prepared as a specially structured Word file and the task is to convert this file into InterBook format. The result of this process is a file with the Textbook in InterBook format which can be served on WWW by the InterBook system.

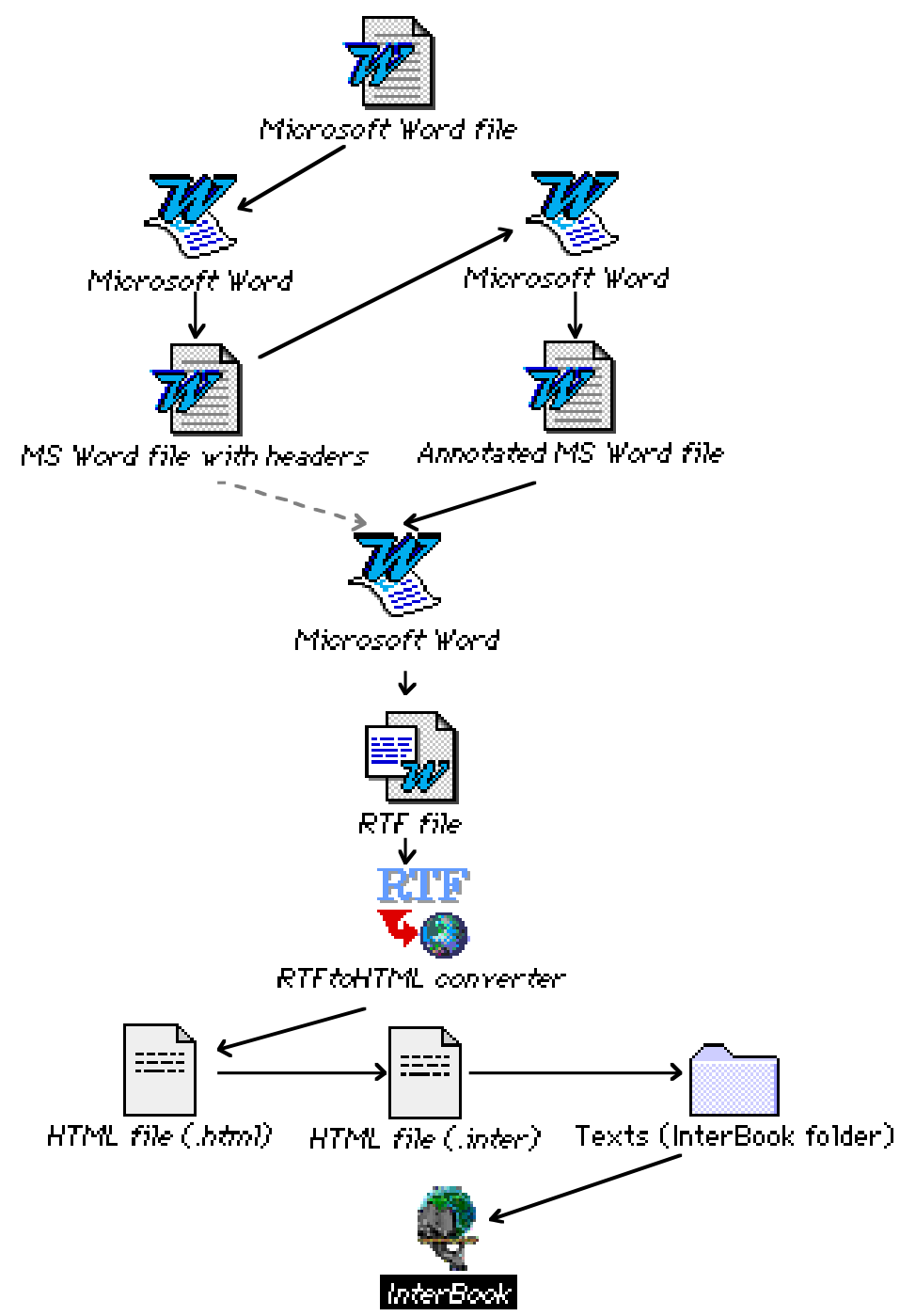

Figure 3. Authoring adaptive ET with InterBook 
InterBook recognizes the structure of the document through the use of headers. It means that the titles of the highest level sections should have a pre-defined text style "Header 1", the titles of its subsections should have a pre-defined paragraph style "Header 2", and so forth. The title of the textbook should have paragraph style "Title". The result of this step will be a properly structured MS Word file.

The second step in the authoring process then involves concept-based annotation of the Electronic Textbook (ET) to let InterBook know which concepts stand behind each section. This knowledge allows InterBook to help the reader of the ET in several ways, and the result of this step is an annotated structured MS Word file.

An annotation is a piece of text of special style and format inserted at the beginning of each section (between the section header and the first paragraph). Annotations have special character style (hidden + shadowed) which are not visible in the text window to the reader of the ET. For each unit the author provides a set of outcome and background concepts. In this way, each section is annotated with a set of prerequisite concepts (or terms which exist in other sections which should be read before the current section), and a set of outcome concepts (terms which will be assumed known once the reader has visited the section). The format for the outcome annotation is: (out: concept-name1, concept-name2, etc.) and the format for the background annotation is: (pre: concept-name1, concept-name2, etc.).

Once the annotations are complete the file is saved in RTF format. The RTFtoHTML program (Hector, 1998) with some special settings is used to convert the ET into HTML format. Then the ".html "extension on the file is manually altered to ".inter" so that it can be recognized by the Interbook system.

Lastly, when the InterBook server starts, it parses all interbook files in its "Texts" folder (i.e. all files with extension .inter) and translates it into the list of section frames. Each unit frame contains the name and type of the unit, its spectrum, and its position in the original HTML file. The obtained LISP structure is used by InterBook to serve all the available textbooks on WWW providing the advanced navigation and adaptation features. The content which is presented to the student is generated on-the-fly using the knowledge about the textbook, the student model, and HTML fragments extracted from the original HTML file. These features of InterBook are based on the functionality of the Common Lisp Hypermedia Server (Mallery, 1994).

As we can see, our tool seriously simplifies the design of adaptive ET on WWW for the authors who use the approach presented in section 2. It provides full support in preparation and serving an ET for the authors who know only how to use the MS Word text processor. An advanced used who have some knowledge of HTML and LISP programming can use our tool more flexibly. For example, an author can bypass step 1 and 2 by preparing the textbook directly in HTML format with annotations provided as specially formatted comments. The author can also replace server response functions and HTML generating functions to implement different structure and different "look and feel" of the be requested by a unique URL. As we mentioned above, to enable the server to respond to a particular URL, this URL has to be associated to a response function implemented in LISP which has to generate an HTML page on the fly as an adaptive response. 


\section{Conclusion}

In this paper we have argued that the progress in Web-based education is related to the development of adaptive courseware which can accommodate users with very different backgrounds, prior knowledge of the subject and learning goals and which can guide the user adaptively through the course. A special role in this process is to be played by authoring tools for the development of adaptive courseware. The main goal of the paper is to present an example of such a tool, namely the InterBook system. We have provided some details about interface, implementation and authoring in Interbook.

We should also briefly mention some results of evaluation studies of the InterBook approach and the InterBook system which we were unable to discuss in this paper due to space limitations. The InterBook adaptive courseware approach was implemented and evaluated in several systems. Empirical studies with ISIS-Tutor (Brusilovsky \& Pesin, 1998) and ELM-ART (Weber \& Specht, 1997) have shown that this approach is really supportive. In particular, the ELM-ART study demonstrated that both adaptive guidance and adaptive navigation support based on this approach are efficient. Adaptive guidance provides significant assistance for novices while adaptive navigation support provides significant assistance for more experienced users. Our recent evaluation of InterBook (Brusilovsky \& Eklund, 1998) shows also that InterBook adaptation mechanisms work quite well. Our positive experiences with InterBook give us hope that InterBook could be used as a model for creating more powerful authoring tools for development adaptive Web-based education.

\section{References}

Brusilovsky, P. (1995) Adaptive learning with WWW: The Moscow State University Project. In: P. Held and W. F. Kugemann (eds.) Telematics for Education and Training. Amsterdam: IOS, pp. 252-255.

Brusilovsky, P. and Eklund, J. (1998) A study of user-model based link annotation in educational hypermedia. Journal of Universal Computer Science, in press.

Brusilovsky, P., Eklund, J., and Schwarz, E. (1997) Adaptive Navigation Support in Educational Hypermedia on the World Wide Web. In: Proceedings of INTERACT97, The 6th IFIP World Conference on Human-Computer Interaction, Sydney, Australia, 14-18 July, 1997. New York: Chapman \& Hall, pp. 278-285.

Brusilovsky, P., Nakabayashi, K., and Ritter, S. (eds.) (1997), Proceedings of the Workshop "Intelligent Educational Systems on the World Wide Web" at AI-ED'97, 8th World Conference on Artificial Intelligence in Education. Kobe, Japan: ISIR. http://www.contrib.andrew.cmu.edu/ plb/AIED97_workshop/

Brusilovsky, P. and Pesin, L. (1998) Adaptive Navigation Support in Educational Hypermedia: An Evaluation of the ISIS-Tutor. Journal of Computing and Information Technology, in press.

Brusilovsky, P., Schwarz, E., and Weber, G. (1996) ELM-ART: An intelligent tutoring system on World Wide Web. In: C. Frasson, G. Gauthier and A. Lesgold (eds.) Proceedings of Third International Conference on Intelligent Tutoring Systems, ITS-96, Montreal, June 12-14, 1996. Berlin: Springer Verlag, pp. 261-269. 
Calvi, L. and De Bra, P. (1997) Using dynamic hypertext to create multi-purpose textbooks. In: T. Müldner and T. C. Reeves (eds.) Proceedings of ED-MEDIA/ED-TELECOM'97 - World Conference on Educational Multimedia/Hypermedia and World Conference on Educational Telecommunications, Calgary, Canada, June 14-19, 1997, AACE, pp. 130-135.

CourseInfo (1997) Interactive Learning Network, http://courses.lightlink.com/web/index.htm

Docent Software Inc. (1997) IBTauthor, http://ibt.testprep.com/

Eklund, J. and Sawers, J. (1996) Customising Web-based course delivery in WEST® with navigation support. Proceedings of WebNet'96, World Conference of the Web Society, San Francisco, CA, October 15-19, 1996, pp. 534-535.

Eliot, C., Neiman, D., and Lamar, M. (1997) Medtec: A Web-based intelligent tutor for basic anatomy. In: S. Lobodzinski and I. Tomek (eds.) Proceedings of WebNet'97, World Conference of the WWW, Internet and Intranet, Toronto, Canada, November 1-5, 1997, AACE, pp. 161-165.

Goldberg, M. W., Salari, S., and Swoboda, P. (1996) World Wide Web - course tool: An environment for building WWW-based courses. Proceedings of Fifth International World Wide Web ConferenceComputer Networks and ISDN Systems 28, 1219-1231, http://www5conf.inria.fr/fich_html/papers/P29/Overview.html.

Graham, C. R. and Trick, T. N. (1997) An innovative approach to asynchronous learning using Mallard: Application of Java applets in a freshman course. In: Proceedings of FIE'97, Frontiers in Education Conference, Pittsburgh, PA, November 5-8, 1997, pp. 238-244.

Harasim, L., Calvert, T., and Groenboer, C. (1997) Virtual-U: A Web-based system to support collaborative learning. In: B. H. Khan (ed.) Web Based Instruction. Englewood Cliffs, New Jersey: Educational Technology Publications, pp. 149-158.

Hector, C. (1998) RTFtoHTML 3.9, http://www.sunpack.com/RTF/

Hubler, A. W. and Assad, A. M. (1995) CyberProf: An Intelligent Human-Computer Interface for Asynchronous Wide Area Training and Breakching. In: Proceedings of 4th International World Wide Web Conference, Boston, USA, December 11-14, 1995, http://www.w3.org/pub/Conferences/WWW4/Papers/247/.

Interactive Factory (1997) CHALK, http://chalk.ifactory.com/

Kay, J. and Kummerfeld, R. J. (1994) An individualised course for the C programming language. In: Proceedings of Second International WWW Conference, Chicago, IL, 17-20 October, 1994, http://www.ncsa.uiuc.edu/SDG/IT94/Proceedings/Educ/kummerfeld/kummerfeld.html.

Lai, M.-C., Chen, B.-H., and Yuan, S.-M. (1995) Toward a new educational environment. In: Proceedings of 4th International World Wide Web Conference, Boston, USA, December 1114, 1995, http://www.w3.org/pub/Conferences/WWW4/Papers/238/.

liveText Publishing (1997) MISK, http://www.misk.com/

Lotus (1997) LearningSpace 2.0, http://www.lotus.com/products/learningspace.nsf

madDuck Technologies (1997) Web Course in a Box (WCB), http://www.madduck.com/wcbinfo/wcb.html

Mallery, J. C. (1994) A Common LISP hypermedia server. In: Proceedings of the First International Conference on the World-Wide Web, May 25, 1994. 
Nakabayashi, K., Maruyama, M., Kato, Y., Touhei, H., and Fukuhara, Y. (1997) Architecture of an intelligent tutoring system on the WWW. In: B. d. Boulay and R. Mizoguchi (eds.) Proceedings of AI-ED'97, World Conference on Artificial Intelligence in Education, Kobe, Japan, 18-22 August 1997. Amsterdam: IOS, pp. 39-46.

Osin, L. (1976) SMITH: How to produce CAI course without programming. International Journal on the Man-Machine Studies 8, 207-241.

Routen, T. W., Graves, A., and Ryan, S. A. (1997) Flax: Provision of interactive courseware on the Web. In: D. Janetzko, B. Batinic, D. Schoder, M. Mattingley-Scott and G. Strube (eds.) Proceedings of Cognition and the Web '97, pp. 149-157, http://www.cms.dmu.ac.uk/coursebook/flax/.

Specht, M., Weber, G., Heitmeyer, S., and Schöch, V. (1997) AST: Adaptive WWW-Courseware for Statistics. In: P. Brusilovsky, J. Fink and J. Kay (eds.) Proceedings of Workshop "Adaptive Systems and User Modeling on the World Wide Web" at 6th International Conference on User Modeling, UM97, Chia Laguna, Sardinia, Italy, June 2, 1997, pp. 91-95, http://www.contrib.andrew.cmu.edu/ plb/UM97_workshop/Specht.html.

Thimbleby, H. (1997) Gentler: a tool for systematic web authoring. In S. B. Shum and C. McKnight (eds.), International Journal on Human-Computer Studies 47 (1).

Vassileva, J. (1997) Dynamic Course Generation on the WWW. In: B. d. Boulay and R. Mizoguchi (eds.) Proceedings of AI-ED'97, 8th World Conference on Artificial Intelligence in Education, Kobe, Japan, 18-22 August 1997. Amsterdam: IOS, pp. 498-505.

WBT Systems (1997) TopClass, http://www.wbtsystems.com/

Weber, G. and Specht, M. (1997) ELM-ART. In: A. Jameson, C. Paris and C. Tasso (eds.) Proceedings of 6th International Conference on User Modeling, Chia Laguna, Sardinia, Italy, June 2-5, 1997. Wien: Springer-Verlag, pp. 289-300.

\section{Acknowledgments}

Part of this work was supported by an Alexander von Humboldt-Stiftung Fellowship and James S. McDonnell Foundation grant to the first author.

\section{Information about authors}

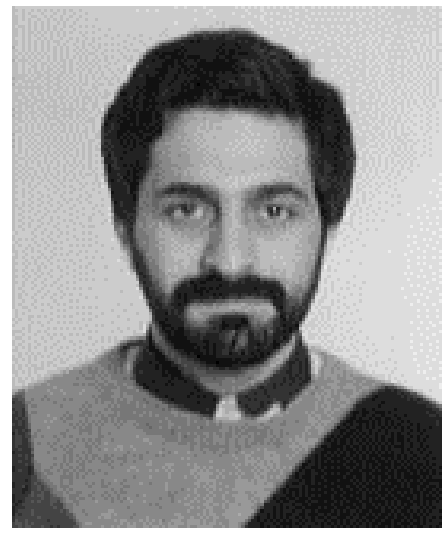

Dr. Peter Brusilovsky is a Visiting Research Scientist at Carnegie Mellon University, School of Computer Science. He received his Ph.D. degree in Computer Science from the Moscow State University in 1987. His research interests includes adaptive interfaces, adaptive hypermedia, and student and user modelling, application of AI technologies to education, and advanced Web-based applications. He authored some 100 technical papers, and edited several books on computer use in education, hypermedia, and multimedia. He is a board member for several journals and member of program committee for a number of international conferences. He is a member of Artificial Intelligence in Education Society and ACM 


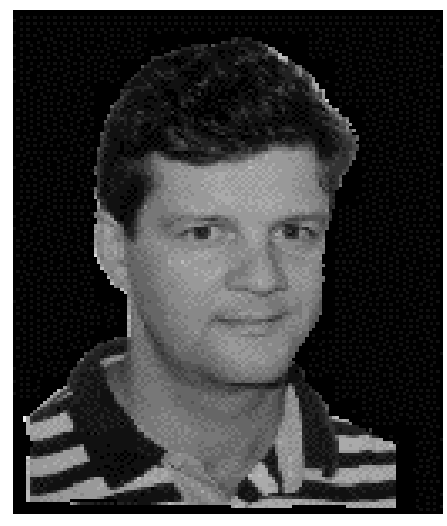

John Eklund is a lecturer in educational computing at the University of Technology, Sydney. He publishes in the area of adaptive navigation support in educational hypermedia, the use of communication technologies in higher education, and computers in schools. He is a member of AACE and serves on a number of journal editorial panels and conference program committees.

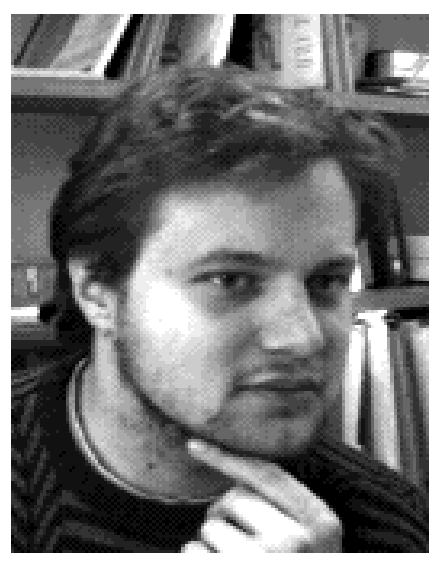

Elmar Schwarz is a student in computer science at the University of Karlruhe, Germany. His interests in artificial intelligence and machine learning led him early towards cognitive sciences in general. Recently, his research carried out in the ACT-group of the Carnegie Mellon University and ELM-group of the University of Trier was focused on intelligent tutoring. In this context he contributed to influential developments in Intelligent Tutoring Systems on the WWW, like ELM-ART and InterBook. 